\title{
Implante de marcapasso endocárdico transatrial concomitante a cirurgia com circulação extracorpórea
}

\author{
Roberto COSTA*, Noedir A. G. STOLF ${ }^{\star}$, Amanda D’ANGELIS* $^{*}$, Geraldo VERGINELLI ${ }^{\star}$, Adib D. JATENE*
}

\section{RBCCV $44205-242$}

COSTA, R.; STOLF, N. A. G.; D'ANGELIS, A.; VERGINELLI, G.; JATENE, A. D. - Implante de marcapasso endocárdico transatrial concomitante a cirurgia com circulação extracorpórea. Rev. Bras. Cir. Cardiovasc., 9(3): $141-145,1994$

RESUMO:Fundamento: A utilização de eletrodos epimiocárdicos permanentes tem sido progressivamente abandonada pelos piores resultados que essa técnica apresenta quando comparada aos eletrodos transvenosos. Implante de eletrodos endocárdicos transtorácicos transatriais tem sido relatado como alternativa ao implante epicárdico em situaçōes especiais. O implante de marcapasso permanente associado a cirurgia cardiaca a céu aberto é uma situação especial onde têm sido utilizados eletrodos epicárdicos no mesmo tempo operatório, ou eletrodos transvenosos em tempos operatórios distintos.

Objetivo: Propor o uso da estimulaçăo transatrial em casos de concomitância de implante de marcapasso e cirurgia cardíaca a céu aberto, e apresentar nossa experiência em 6 pacientes operados.

Casuística e Métodos: De julho de 83 a agosto de 94,6 pacientes com idade variando de 5 a 64 anos, 4 do sexo masculino e 2 do sexo feminino, foram submetidos a cirurgia cardiaca a céu aberto para substituição da valva aórtica (4), resseç̧ão de aneurisma do ventrículo esquerdo por doença de Chagas (1) e atrioseptoplastia e comissurotomia pulmonar (1 paciente). A técnica operatória consistiu em estabelecer a circulação extracorpórea com drenagem venosa por cavas separadas e, durante parada cardioplégica, corrigir o defeito cardíaco e implantar, no mesmo ato, os cabos-eletrodo através de atriotomia direita. Todos os 6 pacientes receberam eletrodos atriais e somente 4 foram submetidos a implante do cabo ventricular. Os geradores de pulso foram implantados na região infraclavicular dos pacientes homens adultos, na região submamária da mulher e na parede abdominal da criança.

Resultados: As medidas elétricas intra-operatórias mostraram excelentes condiçōes de estimulaçāo e sensibilidade, não ocorrendo complicaçōes hospitalares. No seguimento, realizado de 4 a 137 meses, não foram observadas complicaçōes relacionadas ao sistema de estimulação.

Conclusōes: Os autores concluem que o implante de marcapasso endocárdico transatrial pode representar uma boa opção para se evitar o implante epicárdico ou dois procedimentos independentes quando a estimulação cardíaca permanente estiver associada a cirurgia cardiaca a céu aberto..

DESCRITORES: marcapassos cardiacos, cirurgia; estimulação cardiaca artificial, marcapassos.

Trabalho realizado no Instituto do Coração do Hospital das Clínicas da Faculdade de Medicina da Universidade de São Paulo. São Paulo, SP, Brasil. Recebido para publicação em dezembro de 1993.

* Do Instituto do Coração do Hospital das Clínicas da Faculdade de Medicina da Universidade de São Paulo.

Endereço para correspondência: Roberto Costa, Av. Dr. Eneas de Carvalho Aguiar, 44. Divisão Cirúrgica. 05403-100 São Paulo, SP, Brasil. 
COSTA, R.; STOLF, N. A. G.; D'ANGELIS, A.; VERGINELLI, G.; JATENE, A. D. - Implante de marcapasso endocardiaco transatrial concomitante a cirurgia sob circulação extracorpórea. Rev. Bras. Cir. Cardiovasc., 9 (3): 141-145 , 1994

\section{INTRODUÇÃO}

A superioridade dos resultados obtidos com eletrodos de marcapasso permanente implantados pela via endocárdica tem sido demonstrada pelos menores limiares crônicos de estimulação, bem como pela menor incidência de perda de comando por más condições da interface eletrodo-miocárdio ou fratura de condutor 2,6,7. Esses achados são ainda mais expressivos quando analisamos os resultados de estimulação pediátrica, onde o fator crescimento também interfere significativamente no comportamento dos eletrodos 2, 7.

Com o intuito de permitir o implante endocavitário em situaçōes excepcionais, alguns autores têm utilizado abordagens transtorácicas com introdução do eletrodo através da parede atrial $2,5,8$.

A concomitância entre implante de marcapasso permanente e tratamento cirúrgico sob circulação extracorpórea representa uma condição especial que tem sido relatada como ocorrendo em $2,1 \%$ a $3,0 \%$ dos casos de cirurgia cardíaca 3,4 . Nesses pacientes duas condutas distintas têm sido adotadas nos diferentes Serviços: a associação dos dois procedimentos em apenas um tempo cirúrgico, utilizandose eletrodos epimiocárdicos, ou a realização de dois procedimentos isolados com a utilização da via endocárdica para o implante do marcapasso ${ }^{3}$.

O nosso objetivo é propor o implante de marcapasso endocavitário transatrial, quando exista concomitância com cirurgia cardíaca sob circulação extracorpórea, e relatar a experiência do Instituto do Coração do Hospital das Clínicas da Faculdade de Medicina da Universidade de São Paulo.

\section{CASUÍSTICA E MÉTODOS}

Foram estudados 6 pacientes com idade que variou de 5 a 64 anos (média $=33,5$ ); o sexo era masculino em 4 e feminino em 2 pacientes.

A indicação de tratamento cirúrgico foi decorrente de disfunção da valva aórtica em 4 , comunica- ção interatrial com estenose pulmonar valvar em 1 e aneurisma do ventrículo esquerdo em outro paciente. Quanto ao implante de marcapasso ele foi indicado por bloqueio atrioventricular avançado em 4 pacientes e doença do nó sinoatrial em 2. A causa do distúrbio elétrico foi atribuída a valvopatia em 3 pacientes, a seqũela de cirurgia prévia em 1 , a doença de Chagas em 1 e congênito em 1 paciente (Tabela 1).

O procedimento cirúrgico realizado foi substituição da valva aórtica em 4, atrioseptoplastia com comissurotomia pulmonar em 1 aneurismectomia do ventrículo esquerdo em outro paciente (Tabela 2).

O modo de estimulação utilizado foi o atrioventricular universal em 5 e o atrial de demanda em 1 paciente. Um dos pacientes recebeu implante epimiocárdico ventricular, uma vez que, no momento do implante, as cânulas venosas já haviam sido retiradas (Tabela 2 ).

\section{Descrição da Técnica}

Sob anestesia geral endovenosa, os pacientes foram submetidos a toracotomia longitudinal mediana, com esternotomia e instalação de circulação extracorpórea total, para permitir a correção do defeito cardíaco. Durante a preparação para a circulação extracorpórea, as veias cavas superior e inferior foram canuladas separadamente, para permitir a abertura do átrio direito.

Após a correção do defeito que exigia o uso da circulação extracorpórea com o átrio direito aberto $e$, ainda, com a aorta pinçada e o coração parado, realizou-se a passagem dos cabos-eletrodos por contra-abertura na parede atrial. A fixação do eletrodo atrial à parede do átrio direito ou à aurícula direita era reforçada por um ponto de Prolene 6-0. O implante do eletrodo no ventrículo direito era feito sob visão direta, em área de tecido muscular viável, também reforçado com um ponto de Prolene 6-0,

TABELA 1

DADOS CLINIICOS E PESSOAIS DOS PACIENTES

\begin{tabular}{cccccc}
\hline$N^{\circ}$ & IDADE & SEXO & CARDIOPATIA & DISTÚRBIO DA CONDUÇÃO & ETIOLOGIA \\
\hline 1 & 35 & $\mathrm{~F}$ & Aneurisma do VE & Brad. Sinusal Sint. & Doença de Chagas \\
2 & 5 & $\mathrm{~F}$ & CIA + EPV & BAVT & Congênita \\
3 & 26 & $\mathrm{M}$ & Dupla Disfunção Aórtica & BAVT & Valvopatia \\
4 & 25 & $\mathrm{M}$ & Insufic. Aórtica & BAVT & Seqüela Cirúrgica * \\
5 & 46 & $\mathrm{M}$ & Dupla Disfunção Aórtica & Balvopatia \\
6 & 64 & $\mathrm{M}$ & Insuficiência Aórtica & Sindr. Bradi-Taqui & Valvopatia \\
\hline
\end{tabular}

* Correçăo de estenose subaórtica por anel fibroso e plástica da valva aórtica prévias. 
COSTA, R.; STOLF, N. A. G.; D'ANGELIS, A.; VERGINELLI, G.; JATENE, A. D. - Implante de marcapasso endocardiaco transatrial concomitante a cirurgia sob circulação extracorpórea. Rev. Bras. Cir. Cardiovasc., 9 (3): 141-145, 1994

TABELA 2

DADOS OPERATÓRIOS

\begin{tabular}{cccccc}
\hline No & CORREÇÃO CIRÚRGICA & MODO & LOCALDO GERADOR & LOCAL ELETRODOA & LOCALELET.V \\
\hline 1 & Aneurismectomia VE & AAI & SubmamáriaE & Aurícula Direita & Não \\
2 & Fech. CIA + Com. Pulm. & DDD & Fossa llíaca E & Aurícula Direita & Diagragmática VD \\
3 & Troca Aórtica (mecânica) & DDD & Infraclavicular E & AD Alto & VE (epicárdico) \\
4 & Troca Aórtica (mecânica) & DDD & InfraclavicularE & AD Baixo & Septal VD \\
5 & Troca Aórtica (biol.) & DDD & InfraclavicularE & AD Alto & Septal VD \\
6 & Troca Aórtica (biol.) & DDD & InfraclavicularE & AD Alto & Septal VD \\
\hline
\end{tabular}

utilizando-se indistintamente eletrodos de fixação ativa ou passiva. Finalmente; o átrio direito era fechado.

Após a recuperação dos batimentos cardíacos e a interrupção da circulação extracorpórea, antes da retirada das cânulas arterial e venosas, eram realizadas as medidas dos parâmetros eletrofisiológicos: limiar de excitabilidade, resistência e amplitude das ondas $\mathrm{P}$ e R.

O local escolhido para a feitura da loja do gerador foi a região intraclavicular esquerda, no caso dos adultos, e a fossa ilíaca esquerda para a criança, em todos os casos visando facilitar o acesso venoso futuro. Em uma paciente, por razão estética, o gerador de pulsos foi implantado na região submamária esquerda. Os eletrodos eram passados através do gradeado costal ou reborda costal, o gerador conectado aos cabo-eletrodos e fixado à musculatura adjacente.

A retirada das cânulas, a drenagem do mediastino e o fechamento das incisões seguiram a rotina do Serviço.

\section{RESULTADOS}

As medições dos parâmetros eletrofisiológicos obtidas no período intra-operatório estão demonstradas na Tabela 3 . Nenhum dos pacientes neces- sitou mudança de local de implante para obtenção de melhores parâmetros.

Não foram observadas complicações intra-hospitalares.

O seguimento tardio, realizado de 4 a 137 meses, com média de 39,5 , mostrou excelente evolução dos eletrodos endocavitários, como se pode verificar na Tabela 2 . O paciente portador de eletrodo epimiocárdico apresentou aumento do limiar progressivo, o que exigiu, 28 meses após, sua substituição por eletrodo ventricular endocárdico implantado através da veia subclávia esquerda.

\section{COMENTÁRIOS}

A evolução tecnológica da estimulação cardíaca artificial propiciou a substituição progressiva dos implantes de marcapasso epimiocárdicos pelos endocavitários. Facilidade técnica e menor risco operatório são fatores que têm determinado a preferência pela via venosa. Tem sido descrito, também, melhor desempenho a longo prazo dos implantes endocavitários pela maior durabilidade $e$ menor limiar de estimulação ${ }^{6,7}$. Em casos de estimulação permanente pediátrica, a incidência de perda de comando com necessidade de reintervenção ou aumentos significativos de limiar têm sido relatados em até $41 \%$ dos marcapassos epimiocárdicos ${ }^{2}$.

TABELA 3

CABOS-ELETRODO UTILIZADOS E PARÂMETROS INTRA-OPERATÓRIOS

\begin{tabular}{|c|c|c|c|c|c|c|c|c|}
\hline \multirow[b]{2}{*}{$N^{2}$} & \multicolumn{4}{|c|}{ ELETRODO ATRIAL } & \multicolumn{4}{|c|}{ ELETRODO VENTRICULAR } \\
\hline & TIPO FIXAÇĀO & $\begin{array}{c}\text { LIMIAR } \\
(V)\end{array}$ & $\begin{array}{c}\text { RESIST } \\
\text { (OHM) }\end{array}$ & $\begin{array}{c}\text { ONDAP } \\
\text { (MV) }\end{array}$ & TIPO FIXAÇÃO & $\begin{array}{c}\text { LIMIAR } \\
(V)\end{array}$ & $\begin{array}{c}\text { RESIST } \\
\text { (OHM) }\end{array}$ & $\begin{array}{l}\text { ONDA R } \\
\text { (MV) }\end{array}$ \\
\hline 1 & Medtronic 6990 & 0,6 & 420 & 2,4 & N. Realizado & N.R. & N.R. & N.R. \\
\hline 2 & Osypka KY611 & 0,9 & 920 & 5,4 & Osypka KY611 & 0,3 & 630 & 20,0 \\
\hline 3 & Osypka KY611 & 0,7 & 800 & 3,8 & Osypka MP411 & 1,1 & 480 & 18,7 \\
\hline 4 & Osypka VY67 & 1,2 & 740 & 2,3 & Osypka MX67 & 0,3 & 410 & 15,3 \\
\hline 5 & Osypka VY67 & 2,2 & 780 & 3,2 & Osypka MX67 & 0,5 & 670 & 14,4 \\
\hline 6 & Osypka MX66 & 1,9 & 401 & 2,8 & Osypka MX66 & 0,3 & 435 & 10,2 \\
\hline
\end{tabular}


COSTA, R.; STOLF, N. A. G.; D'ANGELIS, A.; VERGINELLI, G.; JATENE, A. D. - Implante de marcapasso endocardiaco transatrial concomitante a cirurgia sob circulação extracorpórea. Rev. Bras. Cir. Cardiovasc., 9 (3): 141-145 , 1994

Nos dias atuais, os Serviços especializados em implante de marcapasso têm praticamente abandonado a técnica epicárdica, que fica reservada para casos excepcionais, como a ausência de acesso venoso ou cardiopatias congênitas com comunicação e fluxo sangüíneo de câmaras direitas para cavidades esquerdas, onde existe a possibilidade de tromboembolismo sistêmico.

A concomitância entre cardiopatia que exija correção sob circulação extracorpórea com distúrbios da condução cardíaca que exijam implante de marcapasso, entretanto, suscita discordância com relação à via de acesso para o implante do marcapasso. Nessa eventualidade, que é relatada entre $2,1 \%$ e $3,0 \%$ dos casos 3,4 , alguns Serviços optam pela associação dos dois procedimentos em apenas um tempo cirúrgico, utilizando eletrodos epimiocárdicos, enquanto outros Serviços optam pela realização de dois procedimentos isolados com a utilização da via endocárdica para o implante do marcapasso definitivo.

Nos pacientes onde o acesso transvenoso está contra-indicado, ou quando a via epimiocárdica está dificultada, várias alternativas de implante endocavitário transatrial têm sido relatadas. WESTERMAN \& VAN DEVANTER ${ }^{8}$ descreveram técnica cirúrgica que permite o implante de eletrodo endocárdico através de toracotomia direita pelo $6^{\circ}$ espaço intercostal, com introdução do cabo-eletrodo no átrio direito através de bolsa feita na aurícula, e posicionamente em átrio direito ou ventrículo direito sob o auxílio de radioscopia. HAYES et alii ${ }^{5}$, durante cirurgia com circulação extracorpórea para substituição de valva tricúspide, implantaram apenas o eletrodo atrial endocárdico transatrial, utilizando a via epimiocárdica para o eletrodo ventricular e BYRD \& SCHWARTZ ${ }^{2}$, utilizam toracotomia com ressecção de cartilagem condro-costal ao nível do $3^{\circ}$ ou $4^{\circ}$ espaço intercostal direito e o auxílio de introdutor de cateter para o acesso ao átrio direito, e posicionamento do eletrodo com o auxílio de radioscopia.

A abordagem presentemente relatada evita os inconvenientes da estimulação epimiocárdica, com a vantagem de não necessitar do auxílio da radioscopia. Os resultados obtidos demonstraram excelentes condições de estimulação e sensibilidade.

Acreditamos, entretanto, que alguns cuidados devam ser observados para facilitar o andamento do procedimento: a) definir previamente o distúrbio da condução, a indicação do maracapasso definitivo e o modo de estimulação; b) canular as veias cavas separadamente, para permitir total liberdade ao exame de átrio e ventrículo direitos; c) implantar os eletrodos com o coração parado, escolhendo regiões do miocárdio atrial e ventricular onde não exista fibrose; d) passar o cabo-eletrodo ventricular através da valva tricúspide em ponto que não atrapalhe sua perfeita coaptação; e) implantar o eletrodo atrial próximo ao nó sinoatrial, para otimizar a sensibilidade da onda P; e) manter as cânulas venosas até a confirmação de parâmetros eletrofisiológicos aceitáveis; f) evitar a passagem dos cabos pela regiảo retroesternal, impedindo sua lesão inadvertida em abordagem futura; g) posicionamento do gerador de pulsos em região que facilite eventual acesso venoso futuro.

Embora a possibilidade da obtenção de parâmetros eletrofisiológicos não aceitáveis estivesse sempre presente, o que exigiria restabelecimento da circulação extracorpórea para mudança da posição do eletrodo, este fato não ocorreu em nossos pacientes. Acreditamos que a escolha cuidadosa da região para o implante, conforme analisado, tenha sido determinante nesse sentido.

\section{CONCLUSÃO}

O implante de marcapasso endocavitário transatrial é uma boa opção para o estabelecimento da estimulação cardíaca artificial permanente quando está indicado em paciente que necessite cirurgia cardíaca sob circulação extracorpórea, evitando os inconvenientes da estimulação epimiocárdica ou de dois tempos operatórios independentes. 
COSTA, R.; STOLF, N. A. G.; D'ANGELIS, A.; VERGINELLI, G.; JATENE, A. D. - Transatrial endocardial pacemaker implantation associated to open heart surgery with cardiopulmonary bypass. Rev. Bras. Cir. Cardiovasc., 9 (3): 141-145, 1994.

ABSTRACT: Background: Epimyocardial pacemaker leads has been progressively given-up due to poorer results in comparison to endocardial pacing. Transthoracic transatrial endocardial lead placement has been described to avoid epicardial pacing in special situations. Permanent pacing associated to open heart surgery is a special situation when epicardial leads have been implanted at the same procedure or endocardial leads have been inserted with an independent approach.

Objective: To propose transatrial endocardial pacemaker in cases of concomitance of permanent pacing and open heart surgery and to report our experience with this technique in 6 patients.

Material and Methods: From July 83 to August 94, 6 patients, 5 to 64 years old, 4 male and 2 female, were submitted to open heart surgery for aortic valve replacement (4), Chaga's disease left ventricular aneurysm resection (1) and atrioseptoplasty and pulmonary valve comissurotomy (1 patient). At the same procedure, atrioventricular (5) or atrial (1 case) pacemaker were implanted. The surgical technique consisted in stablishing cardiopulmonary bypass with two separated vena cava canulas and, under cardioplegic arrest, to correct the cardiac defect and to implant the leads through a rigth atriotomy, at the same procedure. All the 6 patiens received atrial endocardial lead while only 4 patients were submitted to ventricular endocardial implantation. The pulse generators were implanted in the infra-clavicular region in the 4 male adults, in the submammary position in the woman and in the abdominal wall in the child.

Results: Intra-operative measurements showed excellent conditions for pacing and sensing. No peroperative complication occurred. After a 4 to 137 months follow-up period no complications related to the pacemaker were observed.

Conclusions: The authors conclude that transatrial endocardial pacemaker implantation may represent a good option to avoid epicardial leads or two independent procedures when permanent pacing is associated to open heart surgery.

DESCRIPTORS: pacemakers, cardiac, surgery; cardiac stimulation, artificial, pacemakers.

\section{REFERÊNCIAS BIBLIOGRÁFICAS}

1 BYRD, C. L. \& SCHANNON, S. - Transatrial implantation of transvenous pacing leads as an alternative to implantation of epicardial leads. PACE, 13 (Parte 2): 1856-1859, 1990.

2 Gillette, P. C.; ZEIGLER, V. I.; WINSLOW, A. T.; KRATZ, J. M. - Cardiac pacing in neonates, infants and preeschool children. PACE, 15 (Parte 2): 20462049, 1992.

3 GAILLARD, D.; LESPINASSE, P.; VANETTI, A. - Cardiac pacing and valvular surgery. PACE, 11 (Parte 2) 2142-2148, 1988.

4 GOLDMAN, B. S.; HILL, T. J.; WEISEL, R. D.; SCULLY, H. E.; MICKLEBOROUGH, L. L.; PYM, J.; BAIRD, R. J. - Permanent cardiac pacing after open-heart surgery: acquired heart disease. PACE, 7 (Parte 1): 367-371, 1984.

5 HAYES, D. L.; VLIETSTRA, R. E.; PUGA, F. J.; SHUB, C. - A novel approach to atrial endocardial pacing. PACE, 12 (Parte 1): 125-130, 1990.

6 HENGLEIN, D.; GILLETTE, P. C.; SHANNON, C.; BURNS, G. - Long-term follow-up of pulse width threshold of transvenous and myo-epicardial leads. PACE, 7 (Parte 2): 203-214, 1984.

7 SHEPARD, R. B.; KIM, J.; COLVIN, E. C.; SLABAUGH, J.; EPSTEIN, A. E.; BARGERON Jr., L. M. - Pacing threshold spikes months and years after implant. PACE, 14 (Parte 2): 1835-1841, 1991.

8 WESTERMAN, G. R. \& VAN DEVANTER, M. D. Transthoracic transatrial endocardial lead placement for permanent pacing. Ann. Thorac. Surg., 43: 445446, 1987. 